\title{
Fibrose Cardíaca e Mudanças Evolutivas na Função Ventricular Esquerda em Pacientes com Cardiopatia Chagásica Crônica
}

\author{
Cardiac Fibrosis and Changes in Left Ventricle Function in Patients with Chronic Chagas Heart Disease \\ João Bosco de Figueiredo Santos, ${ }^{10}$ Ilan Gottlieb, ${ }^{2}$ Eduardo Marinho Tassi, ${ }^{3}$ (ㄴ) Gabriel Cordeiro Camargo, ${ }^{3}$ (1) \\ Jacob Atié, ${ }^{3}$ Sérgio Salles Xavier, ${ }^{1}$ Roberto Coury Pedrosa, ${ }^{3(0)}$ Roberto Magalhães Saraiva ${ }^{10}$ \\ Instituto Nacional de Infectologia Evandro Chagas/Fundação Oswaldo Cruz, ${ }^{1}$ Rio de Janeiro, RJ - Brasil \\ Casa de Saúde São José, ${ }^{2}$ Rio de Janeiro, RJ - Brasil \\ Hospital Universitário Clementino Fraga Filho/Faculdade de Medicina/Universidade Federal do Rio de Janeiro, ${ }^{3}$ Rio de Janeiro, RJ - Brasil
}

\section{Resumo}

Fundamento: A cardiopatia chagásica (CC) é uma condição de progressão lenta, cujo principal achado histopatológico é fibrose.

Objetivos: Avaliar se a fibrose cardíaca aumenta ao longo do tempo e se correlaciona com aumento no tamanho do ventrículo esquerdo (CE) e redução na fração de ejeção (FE) na CC crônica.

Métodos: Estudo retrospectivo que incluiu 20 indivíduos (50\% homens; $60 \pm 10$ anos) com CC crônica que se submeteram a dois exames de ressonância magnética cardíaca (RMC) com realce tardio com gadolínio em um intervalo mínimo de quatro anos entre os exames. Volume, FE e massa de fibrose do ventrículo esquerdo (VE) foram determinados por RMC. Associações da massa de fibrose na primeira RMC com alterações no volume do VE e FE ventricular esquerda na segunda RMC foram testadas por análise de regressão logística. Valores $p<0,05$ foram considerados significativos.

Resultados: Os pacientes foram classificados em: $A(n=13$; alterações típicas de CC no eletrocardiograma e função sistólica global e segmentar do VE normal) e B1 ( $n=7$; alteração na motilidade da parede do VE e FE $\geq 45 \%)$. O tempo

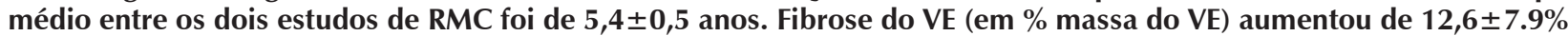
para $18,0 \pm 14,1 \%$ entre os exames de $\operatorname{RMC}(p=0,02)$. A massa de fibrose cardíaca no basal associou-se com uma diminuição > cinco unidades absolutas na FE ventricular esquerda da primeira para a segunda RMC (OR 1,48; IC95\% $1,03-2,13 ; p=0,03)$. A massa de fibrose do VE foi maior e aumentou entre os dois estudos de RMC no grupo de pacientes que apresentaram diminuição na FE entre os testes.

Conclusões: Mesmo pacientes em estágios iniciais da CC apresentam um aumento na fibrose do miocárdio ao longo do tempo, e a presença de fibrose do VE no basal está associada a uma diminuição da função sistólica do VE.

Palavras-chave: Cardiomiopatia Chagásica; Doença de Chagas; Fibrose Endomiocárdica; Disfunção Ventricular Esquerda; Diagnóstico por Imagem; Imagem de Ressonância Magnética/métodos; Eletrocardiografia/métodos.

\footnotetext{
Abstract

Background: Chagas heart disease (CHD) is a slow progressing condition with fibrosis as the main histopathological finding.

Objectives: To study if cardiac fibrosis increases over time and correlates with increase in left ventricular (LV) size and reduction of ejection fraction (EF) in chronic CHD.

Methods: Retrospective study that included 20 individuals (50\% men; 60 10 years) with chronic CHD who underwent two cardiac magnetic resonance imaging (MRI) with late gadolinium enhancement with a minimum interval of four years between tests. $L V$ volume, EF, and fibrosis mass were determined by cardiac MRI. Associations of fibrosis mass at the first cardiac MRI and changes in LV volume and EF at the second cardiac MRI were tested using logistic regression analysis. P values $<0.05$ were considered significant.

Results: Patients were classified as follows: A ( $n=13$; changes typical of CHD in the electrocardiogram and normal global and segmental LV systolic function) and $B 1$ ( $n=7$; LV wall motion abnormality and $E F \geq 45 \%$ ). Mean time between cardiac MRI studies was $5.4 \pm 0.5$ years. LV fibrosis (in \% $L V$ mass) increased from $12.6 \pm 7.9 \%$ to $18.0 \pm 14.1 \%$ between MRI studies $(p=0.02)$. Cardiac fibrosis mass at baseline was associated with decrease in $>5$ absolute units in LV EF from the first to the second MRI (OR 1.48, 95\% CI 1.03-2.13, $p=0.03)$. LV fibrosis mass was larger and increased between MRI studies in the group that presented decrease in LV EF between the tests.
}

Correspondência: Roberto Magalhães Saraiva

Instituto Nacional de Infectologia Evandro Chagas - Fundação Oswaldo Cruz - Av. Brasil, 4365. CEP 21040-900, Rio de Janeiro, RJ - Brasil E-mail: roberto.saraiva@ini.fiocruz.br

Artigo recebido em 12/03/2020, revisado em 18/11/2020, aceito em 27/01/2021

DOI: https://doi.org/10.36660/abc.20200597 
Conclusions: Even patients at an initial stage of CHD show an increase in myocardial fibrosis over time, and the presence of LV fibrosis at baseline is associated with a decrease in LV systolic function.

Keywords: Chagas Cardiomyopathy; Chagas Disease; Endomyocardial Fibrosis; Ventricular Dysfunction, Left; Diagnostic, Imaging; Magnetic Resonance Imaging/methods; Electrocardiography/methods.

Full texts in English - http://www.arquivosonline.com.br

\section{Introdução}

A doença de Chagas é causada pelo protozoário Trypanosoma cruzi, que infecta cerca de 10 milhões de pessoas em todo o mundo $^{1}$ e de 1 a 3 milhões de pessoas no Brasil. ${ }^{2}$ Entre os pacientes com doença de Chagas crônica, 20 a 40\% apresentam a forma cardíaca da doença - cardiopatia chagásica (CC) $-^{2} \mathrm{e}$ cerca de $2 \%$ dos pacientes, a cada ano, irão evoluir da forma indeterminada para a forma cardíaca. ${ }^{3}$

Estudos histopatológicos de amostras de miocárdio obtidas de pacientes com CC revelaram uma cardiomiopatia fibrosante crônica leve, com contínua substituição de fibras miocárdicas por áreas de fibrose e hipertrofia compensatória de miócitos remanescentes, o que poderia estar correlacionado com progressão da CC, remodelamento cardíaco e disfunção sistólica do ventrículo esquerdo (VE). ${ }^{4,5}$

O exame de ressonância magnética cardíaca (RMC) permite a identificação e a quantificação não invasiva de fibrose cardíaca e de anormalidades da motilidade da parede cardíaca, e a avaliação da função sistólica do VE em pacientes com CC. ${ }^{6}$ A massa de fibrose correlaciona-se diretamente com a classe funcional e inversamente com a fração de ejeção do VE. ${ }^{7}$ Ainda, fibrose identificada por RMC associa-se com arritmias ventriculares, ${ }^{8}$ especialmente na presença de duas ou mais áreas contíguas de fibrose transmural. ${ }^{9}$ Estudos longitudinais relataram que a massa de fibrose foi um preditor independente do desfecho combinado de morte cardiovascular, taquicardia ventricular sustentada, ${ }^{10}$ e mortalidade por todas as causas. ${ }^{11}$

Ainda, a RMC pode identificar áreas de fibrose em aproximadamente $20 \%$ dos pacientes com a forma indeterminada de doença de Chagas, ${ }^{7,8}$ e em 43,7\% dos pacientes no estágio A da CC, ${ }^{8}$ com função sistólica de VE normal (segmentar ou global) na ecocardiografia bidimensional. Por outro lado, em pacientes com estágios mais avançados da forma cardíaca, fibrose cardíaca é detectada em 89\%-100\% dos pacientes. ${ }^{7,9}$ Assim, a RMC pode identificar o envolvimento cardíaco precoce na doença de Chagas e a prevalência de pacientes com fibrose cardíaca aumenta com a gravidade das doenças.

Assim, nosso objetivo foi avaliar se a fibrose cardíaca aumenta ao longo do tempo, e se correlaciona com piora da função e geometria do VE. Nós avaliamos retrospectivamente um grupo de pacientes em estágios iniciais da CC que se submeteram a dois exames de RMC com um intervalo mínimo de quatro anos entre os testes.

\section{Métodos}

\section{Amostra do estudo}

Este foi um estudo retrospectivo que incluiu uma amostra de conveniência composta de pacientes adultos com doença de Chagas crônica acompanhados regularmente no ambulatório de doença de Chagas.

Os critérios para a classificação da doença de Chagas seguiram o consenso brasileiro de doença de Chagas: ${ }^{2}$ forma indeterminada (sem evidência de envolvimento cardíaco), forma cardíaca [evidência de alterações típicas de CC no eletrocardiograma (ECG)], forma digestiva (evidência de megacólon ou megaesôfago), ou forma cardiodigestiva. A forma cardíaca foi classificada em estágio A [sem sintomas de insuficiência cardíaca (IC), com alterações isoladas no ECG], estágio B (sem sintomas de IC com disfunção sistólica segmentar e global do VE; B1: fração de ejeção do VE $\geq 45 \%$; B2: fração de ejeção do VE <45\%), estágio C (IC sintomática), ou estágio D (IC terminal).

Foram incluídos no estudo todos os pacientes com CC em estágio A ou B1 submetidos a dois exames de RMC pela técnica do realce tardio com gadolínio, com fibrose cardíaca detectada na primeira RMC, e teste negativo para doença arterial coronariana no teste ergométrico com esteira no momento basal. A maioria dos pacientes incluídos haviam participado de um estudo anterior. ${ }^{8}$

Dados epidemiológicos e clínicos, incluindo comorbidades, sintomas, ecocardiograma, ECG, Holter 24 horas, e exames de sangue, foram obtidos por análise de prontuários médicos.

\section{Ressonância magnética cardíaca (RMC)}

O primeiro exame de RMC foi realizado em um aparelho GE HDxt de 1,5 Tesla (T) (Wakeusha, Wisconsin, EUA), e analisado utilizando o programa ReportCard ${ }^{\circledR} \mathrm{GE}$, versão 3.6, como descrito anteriormente. ${ }^{8} \mathrm{O}$ segundo exame foi realizado em um aparelho Siemens Avanto de 1,5 T (Siemens Healthcare, Alemanha), ou um aparelho Siemens Verio 3,0 $\mathrm{T}$ (Siemens Healthcare, Alemanha). As imagens do VE foram obtidas durante pausa respiratória de 15 segundos, a fim de minimizar artefatos causados por movimentos respiratórios. Imagens do eixo longo e do eixo curto do VE foram obtidas por sequências desencadeadas por ECG nos mesmos locais. Análises da função sistólica do VE e do ventrículo direito foram realizadas por cine-RMC, usando o protocolo de imagens de precessão livre em estado estacionário e o diâmetro diastólico do VE, volume diastólico e sistólico do VE, massa ventricular esquerda, fração de ejeção do VE. Volume diastólico do ventrículo direito, e fração de ejeção foram determinados. Os músculos papilares foram considerados como parte da cavidade do VE para o cálculo do volume e da massa do VE. As imagens foram adquiridas a partir de cortes de $8 \mathrm{~mm}$ e espaçamento de $2 \mathrm{~mm}$ até o ápice do VE. Utilizou-se uma sequência de pulso gradiente eco com inversão-recuperação para avaliação de realce tardio do miocárdio (RTM) em eixos longo e curto do VE. A presença, local, e padrão da fibrose foram determinados qualitativamente. A massa da fibrose foi 
calculada usando o programa ReportCard $® G E$ versão 3.6 na primeira RMC, e o programa CVI42 (Circle Cardiovascular Imaging, Canadá) na segunda RMC. O cálculo da massa de fibrose baseou-se na detecção semiautomática de áreas hiperintensas compatíveis com fibrose no eixo curto nas sequências de RTM. O pesquisador tinha liberdade em delimitar a área de fibrose. Foi adotado um limiar de sinal $\geq 3$ desvios padróes (DPs) acima do sinal médio do miocárdio de referência para se determinar o volume de cicatrização para os dois programas utilizados para o cálculo da massa de fibrose. RTM segmentar foi analisado utilizando-se o modelo de divisão do VE em 17 segmentos. ${ }^{12} \mathrm{O}$ padrão de cicatrização foi classificado como: 1) transmural, se houvesse qualquer área de cicatriz com ocupação $>50 \%$ da espessura da parede, em menos de oito segmentos; 2) focal, se a área de cicatrização não fosse transmural e fosse identificada em menos de oito segmentos; e 3) difusa, se as áreas de cicatrização estivessem presentes em mais de oito segmentos, independentemente da presença de áreas transmurais. ${ }^{10}$ Fibrose em segmentos individuais foi classificada como subendocárdica, medial, subepicárdica, ou transmural.

As análises da primeira RMC foi realizada por dois observadores, e as análises da segunda RMC por dois observadores diferentes, que desconheciam os resultados do primeiro exame.

\section{Análise estatística}

Os cálculos foram realizados utilizando o programa estatístico MedCalc 12.5.0.0. As variáveis contínuas foram expressas em média $\pm \mathrm{DP}$, e as variáveis categóricas em valores absolutos e porcentagens. Todas as variáveis contínuas passaram o teste de normalidade (teste de KolmogorovSmirnov), permitindo o uso de testes paramétricos. Os dados entre a primeira e a segunda RMC foram comparados pelo teste $t$ de Student pareado. A associação entre a massa de fibrose na primeira RMC e alterações na estrutura e função do VE da primeira para a segunda RMC foram testadas por análise de regressão logística. Uma diminuição superior a cinco unidades na fração de ejeção do VE, um aumento $>10 \mathrm{~mL} / \mathrm{m}^{2}$ no volume diastólico do $\mathrm{VE}$, e um aumento $>10 \mathrm{~mL} / \mathrm{m}^{2}$ no volume sistólico do VE foram considerados eventos nessa análise. Valores $\mathrm{p}$ menores que 0,05 foram considerados significativos.

\section{Resultados}

\section{Características dos pacientes}

Vinte pacientes foram incluídos no estudo. Todos apresentavam CC; $65 \%$ no estágio A e 35\% no estágio B1 da doença na primeira RMC. Doença digestiva associada esteve presente em 35\% dos participantes. Observou-se uma igual distribuição de sexos, a maioria dos pacientes nasceu na região nordeste do país, era de etnia branca, apresentava ensino fundamental completo, e era hipertensa (Tabela 1). Nenhum paciente apresentava história de parada cardíaca súbita, sintomas de IC, uso de marcapasso, ou diabetes mellitus. O sintoma mais comum foi palpitações, seguido de pré-síncope, e síncope (Tabela 1). Um paciente teve história de acidente vascular cerebral e outro de ataque isquêmico transitório.
Em relação ao ECG, todos os pacientes estavam em ritmo sinusal, e as principais alterações no ECG foram bloqueio de ramo direito, hemibloqueio anterior esquerdo, e alterações primárias da onda $\mathrm{T}$ (Tabela 1 ). Nenhum participante apresentou bloqueio de ramo esquerdo, baixa voltagem do QRS, ou períodos de inatividade elétrica. Com exceção de um participante, todos os pacientes apresentavam resultados de Holter de 24 horas registrados nos prontuários. Nenhum paciente apresentou taquicardia ventricular sustentada, e somente três apresentaram pausa sinusal maior que dois segundos. Quase 40\% apresentaram uma elevada incidência de contrações ventriculares prematuras, e um quinto apresentou taquicardia ventricular não sustentada nos exames de Holter (Tabela 1). Com exceção de dois pacientes que apresentavam diâmetro sistólico do VE aumentado, e um paciente com diâmetro diastólico do VE aumentado, todos os participantes apresentaram diâmetros do VE e fração de ejeção normais. Metade dos pacientes também apresentaram função diastólica do VE normal (Tabela 1).

Na ocasião da segunda RMC, três pacientes evoluíram do estágio A para o estágio B1; um paciente progrediu do estágio B1 para B2, e um paciente progrediu do estágio B1 para C. Nenhum paciente apresentou algum evento clínico compatível com síndrome coronária aguda durante o acompanhamento.

\section{Ressonância magnética cardíaca}

O tempo médio entre os exames de RMC foi de 5,4 \pm 0,5 anos. A proporção de segmentos do VE com áreas de cicatrização no primeiro e no segundo exames de RMC está apresentada na Figura 1.

O padrão de fibrose foi classificado como focal em 13 pacientes $(65 \%)$ e transmural em sete pacientes $(35 \%)$ na primeira RMC. Sessenta e dois das 340 paredes $(18,2 \%)$ do VE apresentaram fibrose cardíaca com a distribuição: ínfero-lateral basal (55\%), apical (30\%), apical lateral (30\%), apical inferior (25\%), apical anterior (25\%), ínfero-lateral medial (25\%), ínfero-basal (20\%), apical septal (20\%), ântero-lateral medial (20\%), ântero-lateral basal (15\%), ântero-septal basal (10\%), ínfero-septal medial (10\%), anterior basal (5\%), ínfero-septal (5\%), anterior medial (5\%), inferior medial (5\%), e ântero-septal medial (5\%). O padrão de fibrose foi classificado como parede medial em 37 segmentos, transmural em 23 segmentos, subepicardial e parede medial em um segmento, e subendocardial e parede medial em um segmento.

Na segunda RMC, o padrão de fibrose apresentado pelos pacientes foi classificado como focal em 13 pacientes (65\%), transmural em três pacientes (15\%), e difuso em quatro pacientes (20\%). O número de paredes com áreas de fibrose aumentou para 102 de 340 paredes (30\%) e a frequência de paredes com áreas de fibrose foi: ínfero-lateral basal (75\%), inferior basal (50\%), ínfero-lateral medial (45\%), apical lateral (40\%), ântero-lateral basal (35\%), ântero-lateral medial (35\%), ântero-septal basal (30\%), apical (30\%), apical inferior (25\%), apical anterior (25\%), apical septal (25\%), ínfero-septal medial (20\%), inferior medial (20\%), ínfero-septal basal (15\%), anterior medial 
Santos et al.

Fibrose e Função Ventricular Esquerda na Doença de Chagas

Artigo Original

Tabela 1 - Características clínicas e epidemiológicas dos participantes do estudo ( $n=20)$

\begin{tabular}{|c|c|}
\hline Idade, anos & $60,5 \pm 10,4$ \\
\hline Sexo masculino & $10(50 \%)$ \\
\hline \multicolumn{2}{|l|}{ Região de origem } \\
\hline Nordeste & $14(70 \%)$ \\
\hline Sudeste & $5(25 \%)$ \\
\hline Centro-oeste & $1(5 \%)$ \\
\hline \multicolumn{2}{|l|}{ Etnia } \\
\hline Caucasiana & $14(70 \%)$ \\
\hline Mista/Parda & $4(20 \%)$ \\
\hline Afro-brasileira & $2(10 \%)$ \\
\hline \multicolumn{2}{|l|}{ Escolaridade } \\
\hline Analfabeto & $2(10 \%)$ \\
\hline Ensino fundamental & $12(60 \%)$ \\
\hline Ensino médio & $6(30 \%)$ \\
\hline \multicolumn{2}{|l|}{ Parâmetros clínicos } \\
\hline \multicolumn{2}{|l|}{ Forma clínica da DC } \\
\hline Cardíaca - Estágio A & $9(45 \%)$ \\
\hline Cardíaca - Estágio B1 & $4(20 \%)$ \\
\hline Cardiodigestiva A & $4(20 \%)$ \\
\hline Cardiodigestiva B1 & $3(15 \%)$ \\
\hline \multicolumn{2}{|l|}{ Sintomas } \\
\hline Pré-síncope & $5(25 \%)$ \\
\hline Síncope & $3(15 \%)$ \\
\hline Palpitações & $7(35 \%)$ \\
\hline Hipertensão & $13(65 \%)$ \\
\hline \multicolumn{2}{|l|}{ Eletrocardiograma } \\
\hline Bloqueio de ramo direito & $14(70 \%)$ \\
\hline Hemibloqueio anterior esquerdo & $14(70 \%)$ \\
\hline Alterações primárias da onda $T$ & $18(90 \%)$ \\
\hline Contração ventricular prematura & $5(25 \%)$ \\
\hline \multicolumn{2}{|l|}{$\begin{array}{l}\text { Holter } 24 \mathrm{~h} \\
\text { Contração ventricular prematura }\end{array}$} \\
\hline$>30 /$ hora & $7(36,8 \%)$ \\
\hline 10-30/hora & $3(15,8 \%)$ \\
\hline$<10 /$ hora & $5(26,3 \%)$ \\
\hline Ausente & $4(21 \%)$ \\
\hline Taquicardia ventricular não sustentada & $4(21 \%)$ \\
\hline \multicolumn{2}{|l|}{ Ecocardiograma } \\
\hline Diâmetro atrial esquerdo, $\mathrm{cm}$ & $3,5 \pm 0,5$ \\
\hline Diâmetro diastólico do VE, cm & $5,1 \pm 0,5$ \\
\hline Diâmetro sistólico do VE, cm & $3,3 \pm 0,5$ \\
\hline Fração de ejeção do VE, \% & $64,9 \pm 7,9$ \\
\hline Aneurisma do VE & $2(10 \%)$ \\
\hline \multicolumn{2}{|l|}{ Função diastólica do VE } \\
\hline Normal & $10(50 \%)$ \\
\hline Relaxamento tardio & $9(45 \%)$ \\
\hline Não determinada & $1(5 \%)$ \\
\hline
\end{tabular}

DC: Doença de Chagas; VE: ventrículo esquerdo; Valores em média \pm DP ou n (\%). 


\section{Segmentos do VE}

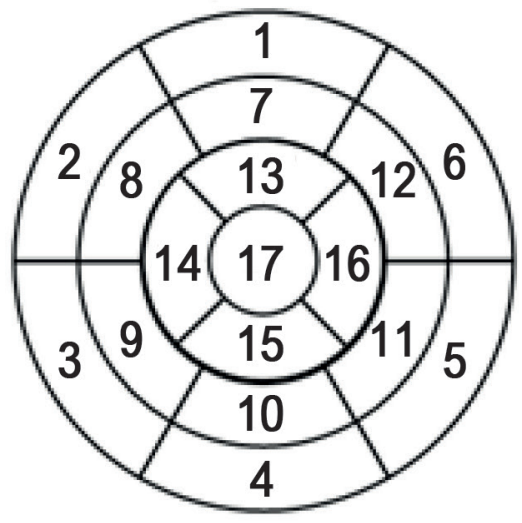

1. Anterior basal

10. Inferiro medial

2. Ântero-septal basal

11. Ínfero-lateral medial

3. Ínfero-septal basal

12. Ântero-lateral medial

4. Ínfero-basal

13. Apical anterior

5. Ínfero-lateral basal

14. Apical septal

6. Ântero-lateral basal

15. Apical inferior

7. Anterior medial

16. Apical lateral

8. Ântero-septal medial

17. Ápice

9. Ínero-septal medial

\section{Proporção (\%) de segmentos do VE com fibrose}

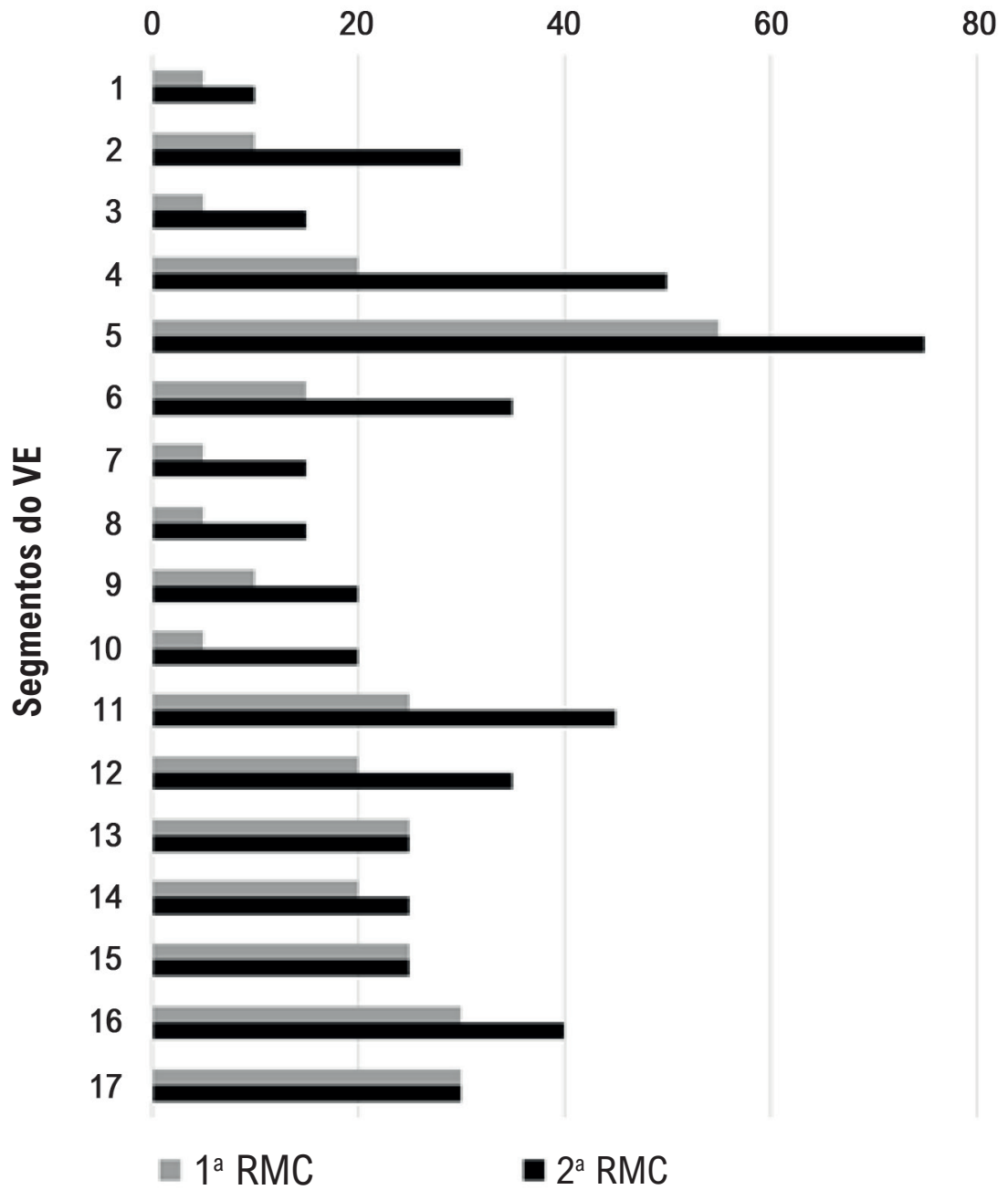

Figura 1 - Proporção de segmentos do ventrículo esquerdo com fibrose na primeira e na segunda ressonância magnética cardíaca segundo modelo de divisão do ventrículo esquerdo em 17 segmentos. 12 Note o aumento na frequência em quase todos os segmentos; VE: ventrículo esquerdo; RMC: ressonância magnética cardíaca. 
(15\%), ântero-septal medial (15\%), e anterior basal (10\%). O padrão de fibrose foi classificado como mesocárdico em 74 segmentos, transmural em 25 segmentos, mesocárdico e subepicárdico em um segmento, e mesocárdico e subendocárdico em dois segmentos.

Em relação à função e tamanho do $V E$, os valores médios do volume sistólico do VE e da massa do VE foram maiores, e a fração de ejeção do VE foi menor no segundo exame de RMC em comparação à primeira RMC. O diâmetro diastólico do VE médio, o volume médio do ventrículo direito, e a fração de ejeção do VE média não mudaram significativamente da primeira para a segunda RMC (Tabela 2).

A massa de fibrose média em porcentagem da massa do VE aumentou $43 \%$ da primeira RMC para a segunda RMC (Figura 2; Tabela 2). Em relação ao padrão de distribuição da fibrose, os pacientes com um padrão transmural apresentaram aumento na massa de fibrose de 19,3 $\pm 6.1 \%$ a $31,4 \pm 14,2 \%, p=0,02$, e aqueles com padrão focal não mostraram aumento significativo na massa de fibrose de $9,0 \pm 6,3 \%$ a $10,8 \pm 7,1 \%, p=0,36$. O número de segmentos do VE com cicatriz aumentou em ambos os grupos: de 38 a 65 (aumento de $71 \%$ ) no grupo com padrão transmural, e de 24 a 37 (aumento de 54,2\%) no grupo com padrão focal de distribuição de fibrose. A massa de fibrose cardíaca aumentou em 11 dos 20 pacientes estudados (Figura 3).

Da primeira para a segunda RMC, 14 pacientes apresentaram uma redução superior a cinco unidades na fração de ejeção do VE, cinco apresentaram um aumento superior a $10 \mathrm{~mL} / \mathrm{m}^{2}$ no volume diastólico do VE e sete apresentaram aumento superior a $10 \mathrm{~mL} / \mathrm{m} 2$ no volume sistólico final do VE. A massa de fibrose cardíaca em porcentagem da massa ventricular esquerda detectada na primeira RMC mostrou uma associação univariada significativa com uma redução maior que cinco unidades na fração de ejeção do VE (OR 1,48, IC95\% 1,03 a $2,13, p=0,03$ ) da primeira para a segunda RMC. Não houve associação significativa univariada ou multivariada entre sexo, idade, massa de fibrose do VE na primeira RMC e mudanças maiores que $10 \mathrm{~mL} / \mathrm{m}^{2}$ no volume diastólico ou sistólico do VE da primeira para a segunda RMC (Tabela 3).
Nós estratificamos os pacientes entre aqueles que apresentaram uma diminuição superior a cinco unidades na fração de ejeção do VE e aqueles que não apresentaram (Figura 3). A massa de fibrose do VE, em porcentagem da massa do VE na primeira RMC foi maior entre os pacientes com diminuição na fração de ejeção do VE que nos pacientes

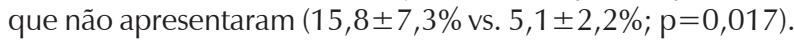
Ainda, a massa de fibrose do VE em porcentagem da massa do VE aumentou da primeira para a segunda RMC somente nos pacientes que apresentaram uma diminuição na fração de ejeção do VE $(15,8 \pm 7,3 \%$ vs. $22,9 \pm 14,2 \%$; $p=0,013$; Figura $3 \mathrm{~A})$. Entre os pacientes que não apresentaram decréscimo na fração de ejeção do VE a massa de fibrose cardíaca em porcentagem da massa do VE, não se alterou da primeira para a segunda RMC $(5,1 \pm 2,2 \%$ vs. $6,7 \pm 2,2 \%$; $p=0,25$; Figura 3B).

\section{Discussão}

A CC é uma doença lenta, implacável, silenciosa caracterizada por uma miocardite fibrosante crônica que culmina em uma miríade de eventos cardiovasculares tais como IC, acidente vascular cerebral, e arritmias fatais. ${ }^{2,4,5}$ Levanta-se a hipótese de que, após um insulto inicial, a lesão cardíaca evolui continuamente até a ocorrência de IC sintomática. ${ }^{13}$ Neste artigo, mostramos, em um grupo de pacientes em estágios iniciais de CC, que a injúria cardíaca causada pela infeção por T. cruzi, estimada pela medida massa de fibrose cardíaca, aumenta ao longo do tempo. Além disso, o grau do dano cardíaco, i.e., da massa de fibrose cardíaca, está associado com diminuição da fração de ejeção do VE.

A fibrose cardíaca é uma marca da CC e um índice prognóstico promissor. Até nosso conhecimento, este é o primeiro estudo a abordar mudanças na massa de fibrose cardíaca e estrutura do VE por meio de RMC em pacientes em estágios iniciais da CC. Nós encontramos um aumento não só na massa de fibrose, como no número de segmentos com fibrose, além de uma piora da função sistólica do VE e um aumento no volume sistólico do VE da primeira para a segunda RMC após um período médio de

Tabela 2 - Comparação da fibrose no ventrículo esquerdo, tamanho e função dos ventrículos direito e esquerdo entre a primeira e a segunda ressonância magnética cardíaca

\begin{tabular}{lccc}
\hline & $\mathbf{1}^{\mathbf{0}} \mathbf{R M C}$ & $\mathbf{2}^{\mathbf{0}} \mathbf{R M C}$ & ${\text { Valor } \mathbf{p}^{\mathbf{a}}}$ \\
\hline Massa de fibrose $(\mathrm{g})$ & $12,4 \pm 9,1$ & $17,9 \pm 16,7$ & 0,03 \\
\hline Massa de fibrose (\% da massa do VE) & $12,6 \pm 7,9$ & $18,0 \pm 14,1$ & 0,02 \\
\hline Diâmetro diastólico do VE, $\mathbf{m m}$ & $53 \pm 4$ & $53 \pm 7$ & 0,90 \\
\hline Volume diastólico do VE, $\mathrm{mL} / \mathrm{m}^{2}$ & $76,6 \pm 19,1$ & $76,8 \pm 21,7$ & 0,94 \\
\hline Volume sistólico do VE, $\mathrm{mL} / \mathrm{m}^{2}$ & $30,5 \pm 13,1$ & $37,9 \pm 17,9$ & 0,004 \\
\hline Fração de ejeção do VE, $\%$ & $61,1 \pm 9,5$ & $52,5 \pm 11,7$ & $<0,0001$ \\
\hline Massa do VE, $\mathbf{g} / \mathrm{m}^{2}$ & $53,9 \pm 11,8$ & $56,5 \pm 12,6$ & 0,008 \\
\hline Volume diastólico do VD, $\mathrm{mL} / \mathrm{m}^{2}$ & $58,9 \pm 14,6$ & $62,0 \pm 15,8$ & 0,11 \\
\hline Fração de ejeção do VD, $\%$ & $56,7 \pm 3,2$ & $56,1 \pm 10,2$ & 0,79
\end{tabular}

VE: ventrículo esquerdo; RMC: ressonância magnética cardiaca; VD: ventrículo direito. aTeste t de Student pareado. 


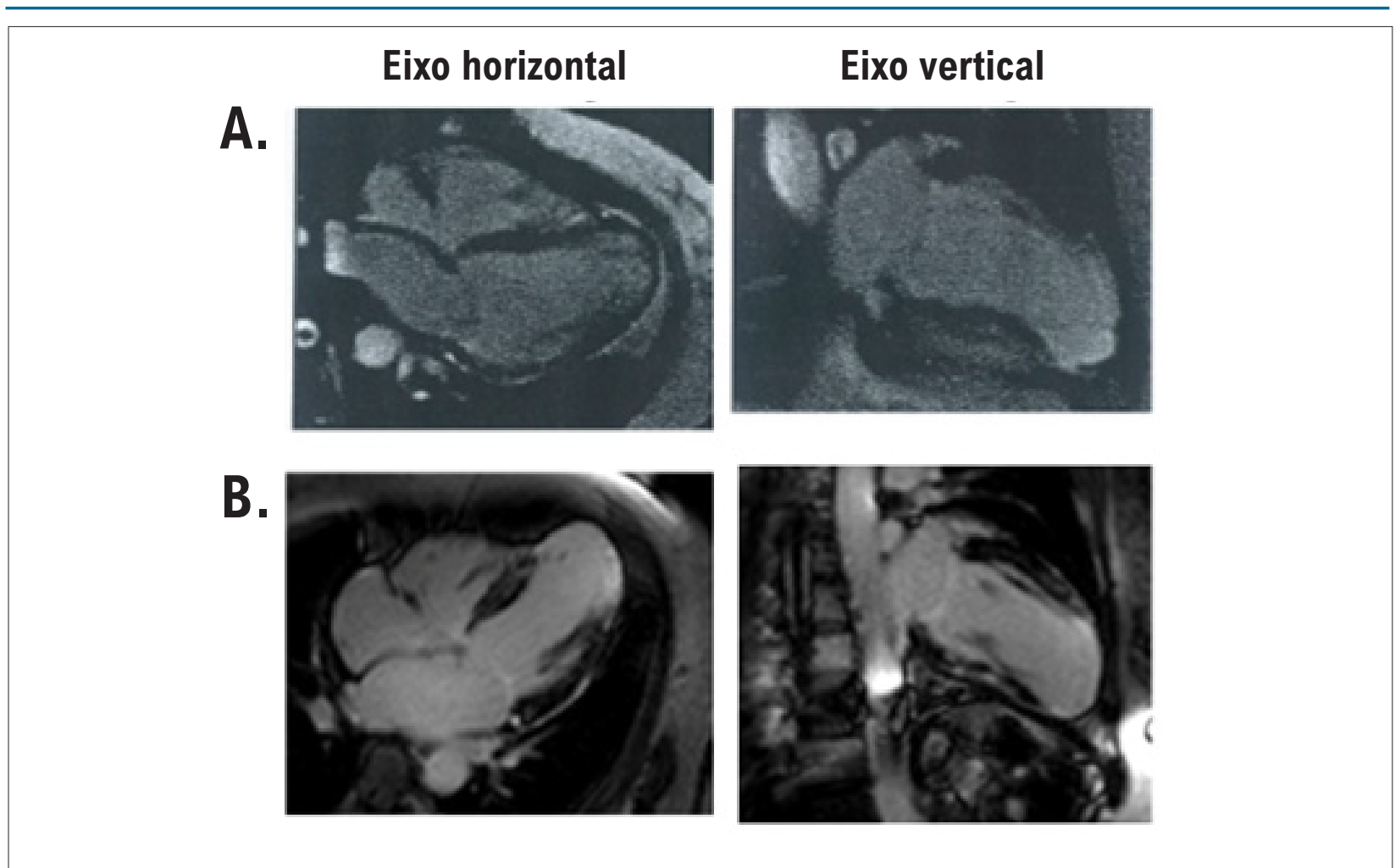

Figura 2 - Ressonância magnética cardíaca de um paciente com alterações progressivas na massa de fibrose. A) Realce tardio do miocárdio nos cortes do eixo horizontal e vertical mostra áreas de fibrose na parede média (áreas brilhantes) nos segmentos mediais do septo, e fibrose transmural em todas as paredes apicais e no ápice. A massa de fibrose do miocárdio foi estimada em 33 gramas. B) Imagens do realce tardio do miocárdio obtidas do mesmo paciente 4,5 anos depois mostram áreas de fibrose a parede medial nos segmentos basais das paredes ínfero-septal, infero-lateral, e ântero-lateral, segmentos mediais das paredes anteriores, e fibrose transmural nos segmentos mediais das paredes ínfero-lateral e ântero-lateral, todas as paredes apicais e ápice. A massa de fibrose do miocárdio foi estimada em 58 gramas.

acompanhamento de cinco anos. O aumento na fibrose do VE ocorreu principalmente no grupo de pacientes com fibrose transmural. Em nosso estudo, fibrose do VE foi associada com diminuição da fração de ejeção do VE ao longo do tempo. A massa de fibrose do VE foi maior na primeira RMC e aumentou da primeira para a segunda RMC somente nos pacientes que apresentaram uma diminuição na fração de ejeção do VE.

Pacientes com doença de Chagas com fibrose cardíaca apresentam menor fração de ejeção do VE e maior volume e massa do $V E$, e maior dimensão do átrio esquerdo que pacientes sem fibrose cardíaca. ${ }^{10,14}$ De fato, há uma forte correlação negativa entre massa de fibrose do VE e fração de ejeção do VE. ${ }^{7,15} \mathrm{Em}$ um estudo anterior de nosso grupo, somente pacientes com fibrose cardíaca apresentaram progressão da doença de Chagas e piora da função do VE medida pela deformação (strain) longitudinal e circunferencial do VE. ${ }^{16}$ Fibrose do miocárdio associou-se independentemente com mortalidade por todas as causas em um estudo retrospectivo, ${ }^{11}$ e com a ocorrência do desfecho combinado de morte cardiovascular e taquicardia ventricular sustentada em um estudo prospectivo. ${ }^{10}$

$\mathrm{Na}$ primeira RMC, fibrose foi mais comumente observada nos segmentos ínfero-lateral e apical, como mostrado anteriormente na doença de Chagas. ${ }^{7-9}$ Após um acompanhamento médio de 5,4 anos, a prevalência de fibrose cardíaca aumentou em quase todos os segmentos, mais pronunciadamente nos segmentos basais. Houve uma piora no padrão de fibrose cardíaca, uma vez que, em quatro pacientes, o padrão de fibrose mudou de transmural para difusa. Isso reforça a natureza progressiva do dano cardíaco causado pela doença de Chagas.

Nossos dados mostraram que a massa de fibrose do miocárdio aumenta ao longo do tempo, o que pode estar relacionado com uma miocardite fibrosante de progressão lenta, com alterações na matriz extracelular que leva à remodelação cardíaca e IC. De fato, as enzimas envolvidas na modulação da matriz extracelular [metaloproteinases (MMP) 2 e 9], presentes na patogênese de várias doenças cardiovasculares, ${ }^{17}$ podem ter um importante papel na patogênese da doença de Chagas. Mudanças no equilíbrio entre MMP e suas atividades inibitórias podem ser importantes para remodelação cardíaca. ${ }^{18} \mathrm{Em}$ camundongos infectados por T. cruzi, tratamento com inibidores de MMP-2/MMP-9 induziu uma redução na inflamação do miocárdio e aumento na sobrevida. ${ }^{19} \mathrm{Em}$ pacientes com doença de Chagas crônica, níveis séricos de MMP-2 e MMP-9 são maiores nos pacientes com as formas indeterminada e cardíaca em comparação a controles. ${ }^{20,21}$ Contudo, os níveis séricos de MMP-2 foram 


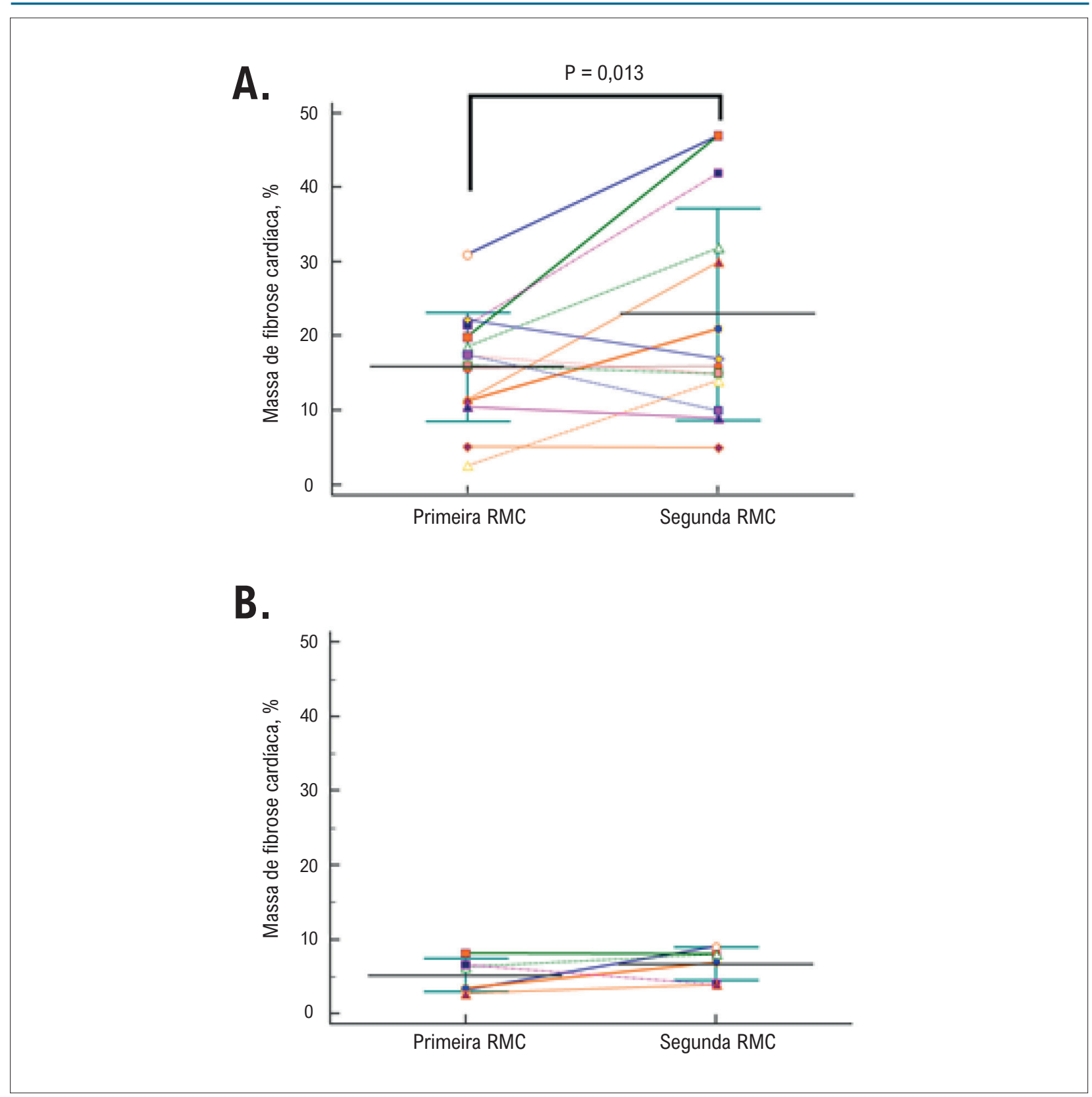

Figura 3 - Mudanças individuais da massa de fibrose, em porcentagem da massa do ventrículo esquerdo (VE) do primeiro exame de ressonância magnética cardiaca (RMC) ao segundo exame de RMC em pacientes que apresentaram uma diminuição na fração de ejeção do VE ao longo do tempo (A) e pacientes que não apresentaram diminuição na fração de ejeção do VE (B). Note que a massa de fibrose do VE é maior no basal e aumentou da primeira para a segunda RMC somente no grupo de pacientes que apresentaram redução na fração de ejeção do VE ao longo do tempo.

mais altos em pacientes com a forma cardíaca que a forma indeterminada, ${ }^{21,22}$ ao passo que os níveis de MMP-9 foram maiores nos pacientes com a forma indeterminada que a forma cardíaca. ${ }^{22}$ Portanto, um equilíbrio entre MMP-2 e MMP-9 parece ser importante na progressão da doença de Chagas. $^{23}$

\section{Limitações}

Limitações deste estudo incluem seu delineamento retrospectivo, pequena amostra de conveniência, e o fato de os exames de RMC terem sido realizados em equipamentos de diferentes fabricantes, e analisados por diferentes especialistas, sem uma avaliação da variabilidade interobservador. No entanto, a concordância entre os observadores para massa de fibrose cardíaca do grupo que realizou a primeira RMC foi excelente, como já demonstrado previamente. ${ }^{8}$ Em um estudo prévio, ${ }^{24}$ a variabilidade interobservador para a técnica de quantificação de fibrose baseada no limiar de sinal versus miocárdio de referência (do inglês signal threshold versus reference myocardium, 


\begin{tabular}{|c|c|c|c|c|c|c|}
\hline & \multicolumn{3}{|c|}{ Associação univariada } & \multicolumn{3}{|c|}{ Associação multivariada } \\
\hline & OR & IC95\% & Valor $\mathrm{p}$ & OR & IC95\% & Valor $p$ \\
\hline \multicolumn{7}{|c|}{ Redução > 5 unidades na fração de ejeção do VE } \\
\hline Sexo masculino & 0,37 & $0,05-2,77-$ & 0,34 & 0,14 & $0,00-15,9$ & 0,42 \\
\hline Idade (anos) & 1,03 & $0,94-1,13$ & 0,47 & 1,37 & $0,89-2,11$ & 0,15 \\
\hline Massa de fibrose do VE, \% & 1,48 & $1,03-2,13$ & 0,03 & 2,27 & $0,87-5,91$ & 0,09 \\
\hline \multicolumn{7}{|c|}{ Aumento $>10 \mathrm{~mL} / \mathrm{m}^{2}$ no volume diastólico do VE } \\
\hline Sexo masculino & 0,58 & $0,07-4,56$ & 0,61 & 0,56 & $0,04-7,65$ & 0,66 \\
\hline Idade (anos) & 1,10 & $0,96-1,27$ & 0,17 & 1,12 & $0,96-1,31$ & 0,15 \\
\hline Massa de fibrose do VE, \% & 1,04 & $0,91-1,18$ & 0,56 & 1,05 & $0,89-1,24$ & 0,57 \\
\hline \multicolumn{7}{|c|}{ Aumento $>10 \mathrm{~mL} / \mathrm{m}^{2}$ no volume sistólico do VE } \\
\hline Sexo masculino & 0,64 & $0,10-4,10$ & 0,64 & 1,03 & $0,07-14,8$ & 0,98 \\
\hline Idade (anos) & 1,13 & $0,97-1,31$ & 0,10 & 1,17 & $0,97-1,40$ & 0,09 \\
\hline Massa de fibrose do VE, \% & 1,06 & $0,94-1,20$ & 0,33 & 1,11 & $0,93-1,33$ & 0,24 \\
\hline
\end{tabular}

VE: ventrículo esquerdo; análise de regressão logística (método Enter).

STRM), que nós usamos para avaliar fibrose do VE, foi -1,2\% (IC95\% -8,8 a 9,2\%). ${ }^{24}$ Em nosso estudo, seis pacientes apresentaram uma diminuição na massa de fibrose no VE em porcentagem da massa do VE, que variou de 1,56 a 7,48\%, todos dentro dos limites do intervalo de confiança de $95 \%$ para a variabilidade interobservador. Por outro lado, dos 11 pacientes que apresentaram um aumento na massa de fibrose do VE, em porcentagem da massa do VE, sete apresentaram um aumento acima do limite do intervalo de confiança de 95\% descrito para a variabilidade interobservador. Três outros pacientes apresentaram uma diferença inferior a um porcento entre os exames. Assim, todos os pacientes com uma diferença entre exames maior que a variabilidade do teste mostraram um aumento na massa de fibrose do VE e corresponderam a 35\% da população estudada. Assim, acreditamos que a variabilidade intraobservador e entre observadores não causou viés em nossos resultados.

Quanto à exclusão de pacientes com doença arterial coronariana prévia, um resultado negativo no teste ergométrico com esteira não exclui a possibilidade de uma oclusão total de um ramo coronariano. No entanto, os pacientes negaram a existência de eventos clínicos prévios compatíveis com síndrome coronariana aguda. Ainda, os pacientes não foram submetidos a um segundo teste ergométrico ou à angiografia coronária antes da segunda RMC para excluir possível doença arterial coronariana. Contudo, nenhum paciente apresentou qualquer evento clínico compatível com doença arterial coronariana durante o acompanhamento do estudo.

\section{Conclusões}

Neste estudo retrospectivo que incluiu pacientes em estágios iniciais da CC, a fibrose miocárdica aumentou ao longo do tempo, e a fibrose do VE no basal associou-se a uma diminuição da função sistólica do VE. Esse importante achado deve ser confirmado em estudos prospectivos. A fibrose cardíaca deve ainda ser mais estudada como um índice prognóstico para a evolução da doença de Chagas e eventos cardiovasculares.

\section{Contribuição dos autores}

Concepção e desenho da pesquisa: Santos JBF, Xavier SS, Pedrosa RC, Saraiva RM; Obtenção de dados: Santos JBF, Gottlieb I, Tassi EM, Camargo GC; Análise e interpretação dos dados: Santos JBF, Atié J, Xavier SS, Pedrosa RC, Saraiva RM; Análise estatística: Santos JBF, Saraiva RM; Redação do manuscrito: Santos JBF, Tassi EM, Camargo GC, Pedrosa RC, Saraiva RM; Revisão crítica do manuscrito quanto ao conteúdo intelectual importante: Santos JBF, Gottlieb I, Tassi EM, Camargo GC, Atié J, Xavier SS, Pedrosa RC, Saraiva RM.

\section{Potencial conflito de interesse}

Não há conflito com o presente artigo.

\section{Fontes de financiamento}

O presente estudo foi parcialmente financiado pela CAPES.

\section{Vinculação acadêmica}

Este artigo é parte de tese de Doutorado de João Bosco de Figueiredo Santos pelo Instituto Nacional de Infectologia Evandro Chagas/Fiocruz.

\section{Aprovação ética e consentimento informado}

Este estudo foi aprovado pelo Comitê de Ética do CEP INI Fiocruz; CEP HUCFF UFRJ sob os números de protocolo 3.146.237 e 11.186.956. Todos os procedimentos envolvidos nesse estudo estão de acordo com a Declaração de Helsinki de 1975, atualizada em 2013. O consentimento informado foi obtido de todos os participantes incluídos no estudo. 


\section{Referências}

1. World Health Organization. (WHO). Chagas Disease (American trypanosomiasis). [Cited in 2020 Apr 12] Available at: http://www.who.int/ mediacentre/factsheets/fs340/en/ . 2017.

2. Dias JC, Ramos AN Jr, Gontijo ED, Luquetti A, Shikanai-Yasuda MA, Coura JR, et al. Brazilian Consensus on Chagas Disease, 2015. Rev Soc Bras Med Trop. 2016;49(Suppl 1):3-60.

3. Sabino EC, Ribeiro AL, Salemi VM, Di Lorenzo OC, Antunes AP, Menezes MM, et al. Ten-year incidence of Chagas cardiomyopathy among asymptomatic Trypanosoma cruzi-seropositive former blood donors. Circulation 2013;127(10):1105-15.

4. Chaves AT, Menezes CAS, Costa HS, Nunes MCP, Rocha MOC. Myocardial fibrosis in chagas disease and molecules related to fibrosis. Parasite Immunol 2019;41(10):e12663.

5. Bonney KM, Luthringer DJ, Kim SA, Garg NJ, Engman DM. Pathology and Pathogenesis of Chagas Heart Disease. Annu Rev Pathol. 2019;14:421-47.

6. Nunes MCP, Badano LP, Marin-Neto JA, Edvardsen T, Fernandez-Golfin C, Bucciarelli-Ducci $\mathrm{C}$, et al. Multimodality imaging evaluation of Chagas disease: an expert consensus of Brazilian Cardiovascular Imaging Department (DIC) and the European Association of Cardiovascular Imaging (EACVI). Eur Heart J Cardiovasc Imaging 2018;19(4):459-60n.

7. Rochitte CE, Oliveira PF, Andrade JM, lanni BM, Parga JR, Avila LF, et al. Myocardial delayed enhancement by magnetic resonance imaging in patients with Chagas' disease: a marker of disease severity. J Am Coll Cardiol 2005;46(8):1553-8.

8. Tassi EM, Continentino MA, Nascimento EM, Pereira BB, Pedrosa RC. Relationship between fibrosis and ventricular arrhythmias in Chagas heart disease without ventricular dysfunction. Arq Bras Cardiol 2014;102(5):456-64.

9. Mello RP, Szarf G, Schvartzman PR, Nakano EM, Espinosa MM, Szejnfeld $D$, et al. Delayed enhancement cardiac magnetic resonance imaging can identify the risk for ventricular tachycardia in chronic Chagas' heart disease. Arq Bras Cardiol 2012;98(5):421-30.

10. Volpe GJ, Moreira HT, Trad HS, Wu KC, Braggion-Santos MF, Santos MK, et al. Left Ventricular Scar and Prognosis in Chronic Chagas Cardiomyopathy. J Am Coll Cardiol 2018;72(21):2567-76.

11. Senra T, lanni BM, Costa ACP, Mady C, Martinelli-Filho M, Kalil-Filho R, et al. Rochitte CE. Long-Term Prognostic Value of Myocardial Fibrosis in Patients With Chagas Cardiomyopathy. J Am Coll Cardiol 2018;72(21):2577-87.

12. Cerqueira MD, Weissman NJ, Dilsizian V, Jacobs AK, Kaul S, Laskey WK, et al. Standardized myocardial segmentation and nomenclature for tomographic imaging of the heart. A statement for healthcare professionals from the Cardiac Imaging Committee of the Council on Clinical Cardiology of the American Heart Association. Circulation. 2002;105(4):539-42.
13. Benchimol-Barbosa PR. Nonlinear mathematical model for predicting long term cardiac remodeling in Chagas' heart disease: introducing the concepts of 'limiting cardiac function' and 'cardiac function deterioration period'. Int J Cardiol. 2010;145(2):219-21.

14. Regueiro A, Garcia-Alvarez A, Sitges M, Ortiz-Perez JT, De Caralt MT, Pinazo $M J$, et al. Myocardial involvement in Chagas disease: insights from cardiac magnetic resonance. Int J Cardiol. 2013;165(1):107-12.

15. Uellendahl M, Siqueira ME, Calado EB, Kalil-Filho R, Sobral D, Ribeiro C, et al. Cardiac Magnetic Resonance-Verified Myocardial Fibrosis in Chagas Disease: Clinical Correlates and Risk Stratification. Arq Bras Cardiol. 2016;107(5):460-6.

16. Gomes VA, Alves GF, Hadlich M, Azevedo CF, Pereira IM, Santos CR, et al. Analysis of Regional Left Ventricular Strain in Patients with Chagas Disease and Normal Left Ventricular Systolic Function. J Am Soc Echocardiogr. 2016;29(7):679-88.

17. Nagase H, Visse R, Murphy G. Structure and function of matrix metalloproteinases and TIMPs. Cardiovasc Res. 2006;69(3):562-73.

18. Spinale FG. Myocardial matrix remodeling and the matrix metalloproteinases: influence on cardiac form and function. Physiol Ver. 2007;87(4):1285-342.

19. Gutierrez FR, Lalu MM, Mariano FS, Milanezi CM, Cena J, Gerlach RF, et al. Increased activities of cardiac matrix metalloproteinases matrix metalloproteinase (MMP)-2 and MMP-9 are associated with mortality during the acute phase of experimental Trypanosoma cruzi infection. J Infect Dis. 2008;197(10):1468-76.

20. Bautista-Lopez NL, Morillo CA, Lopez-Jaramillo P, Quiroz R, Luengas C, Silva SY, et al. Matrix metalloproteinases 2 and 9 as diagnostic markers in the progression to Chagas cardiomyopathy. Am Heart J. 2013;165(4):558-66.

21. Fares RC, Gomes JA, Garzoni LR, Waghabi MC, Saraiva RM, Medeiros NI, et al. Matrix metalloproteinases 2 and 9 are differentially expressed in patients with indeterminate and cardiac clinical forms of chagas disease. Infect Immun. 2013;81(10):3600-8.

22. Clark EH, Marks MA, Gilman RH, Fernandez AB, Crawford TC, Samuels AM, et al. Circulating serum markers and QRS scar score in Chagas cardiomyopathy. Am J Trop Med Hyg. 2015;92(1):39-44.

23. Medeiros NI, Gomes JAS, Correa-Oliveira R. Synergic and antagonistic relationship between MMP-2 and MMP-9 with fibrosis and inflammation in Chagas' cardiomyopathy. Parasite Immunol. 201;39(8):e12446.

24. Fine NM, Tandon S, Kim HW, Shah DJ, Thompson T, Drangova M, et al. Validation of sub-segmental visual scoring for the quantification of ischemic and nonischemic myocardial fibrosis using late gadolinium enhancement MRI. J Magn Reson Imaging. 2013;38(6):1369-76. 